\title{
Propagation of polarized light in birefringent turbid media: A Monte Carlo study
}

\author{
Xueding Wang \\ Lihong V. Wang \\ Texas A\&M University \\ Optical Imaging Laboratory \\ Biomedical Engineering Program \\ 3120 TAMU, College Station, Texas 77843-3120
}

\begin{abstract}
A detailed study, based on a Monte Carlo algorithm, of polarized light propagation in birefringent turbid media is presented in this paper. Linear birefringence, which results from the fibrous structures, changes the single scattering matrix and alters the polarization states of photons propagating in biological tissues. Some Mueller matrix elements of light backscattered from birefringent anisotropic turbid media present unusual intensity patterns compared with those for nonbirefringent isotropic turbid media. This result is in good agreement with the analytic results based on the double-scattering model. Degree of polarization, Stokes parameters, and diffuse reflectance as functions of linearly birefringent parameters based on numerical results and theoretical analysis are discussed and compared in an effort to understand the essential physical processes of polarized light propagation in fibrous tissues. (c) 2002 Society of Photo-Optical Instrumentation Engineers. [DOI: 10.1117/1.1483315]
\end{abstract}

Keywords: birefringence; scattering media; propagation; polarized light; Monte Carlo algorithm.

Paper TP-05 received July 22, 2001; revised manuscript received Mar. 20, 2002; accepted for publication Mar. 29, 2002.

\section{Introduction}

Because of the potential for practical applications, especially in noninvasive medical diagnosis, there has been increasing interest recently in the propagation of polarized light in randomly scattering media. Parameters, such as particle size, particle shape, particle density, the properties of the background around the scattering particle, and the polarization states of incident light, all play important roles in the propagation of light in turbid media. ${ }^{1-3}$ It is widely recognized that the polarization states of light are altered or weakened after photons are scattered by small particles in a medium. At the same time, because the depolarization of light depends on the parameters of the turbid media as well as the polarization state of the incident light, it provides a way to image tumors. For example, Anderson, ${ }^{4}$ Jacques et al., ${ }^{5}$ Demos, and Alfano ${ }^{6}$ studied the utilization of backscattered polarized light for the imaging of tissue structures beneath surface.

It is known in the field of polarimetry that Stokes vectors and Mueller matrices provide complete representations of the polarization properties of light and optical samples, respectively. Hielscher et al. ${ }^{7}$ introduced a Stokes vector/Mueller matrix approach and measured the spatially dependent Mueller matrix for diffusely backscattered light. Cameron et al. ${ }^{8}$ and Rakovic et al. ${ }^{9}$ compared experimental results with a Monte Carlo-simulated 16 Mueller matrix elements of light backscattered from a suspension of polystyrene spheres. They proved that there are only seven independent matrix elements because some symmetric relations are satisfied by the elements of the backscattering matrix. Through theoretical analysis, Rakovic and Kattawar ${ }^{10}$ showed that a double-scattering model can effectively emulate the spatial patterns of backscat-

Address all correspondence to Lihong Wang. Tel: 979-847-9040; Fax: 979-8454450; E-mail: Iwang@tamu.edu tered light obtained from experiments. Yao and Wang ${ }^{11}$ used a time-resolved Monte Carlo technique to present the propagation of polarized light in turbid media. All of the above studies were conducted on isotropic turbid media without considering the birefringent effect on polarizations.

Collagen and muscle fibers are common constituents of many biological tissues. Tissue birefringence results primarily from the linear anisotropy of fibrous structures, which forms the extracellular media. The refractive index of the medium is higher along the length of fibers than that along the cross section. Propagation of polarized light in a birefringent turbid medium is complicated, because both the birefringent and the scattering effects can change the polarization states of light. Recently, measurements of birefringence using optical coherence tomography (OCT) were reported by several research groups, ${ }^{12-16}$ where a low-coherence light source was employed and photons traveling in tissue with matched path lengths were detected for subsurface tissue imaging.

Although the optical characteristics of birefringent tissue is an area of considerable interest in biomedical imaging, no systematic and numerical analysis has been previously made on the polarization change that occurs in multiply scattered photons in birefringent turbid media. This paper describes our detailed analysis, based on a Monte Carlo method, of polarized light propagating in, or backscattered from, birefringent turbid media. In the second section, we will introduce our Monte Carlo algorithm based on Mie theory that we use for tracing the photons in birefringent turbid media. A doublescattering mode ${ }^{10}$ that can be used to predict the backscattering Mueller matrix will be presented for comparison in the third section. The fourth section will describe simulated backscattering matrix patterns based on the Monte Carlo method.

1083-3668/2002/\$15.00 @ 2002 SPIE 


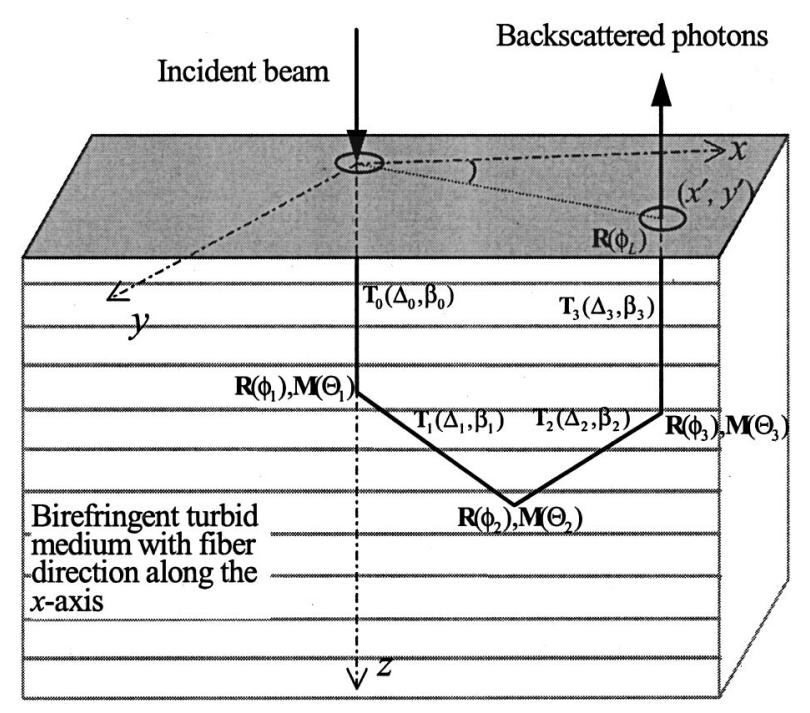

Fig. 1 Geometry of a multiple scattering event in a linearly birefringent turbid medium.

The diffuse reflectance, the Stokes parameters, and the degree of polarization (DOP) of backscattered photons from turbid media with birefringence will also be analyzed. The final section will present the conclusions.

\section{Monte Carlo Algorithm}

The following Monte Carlo analysis of multiple scattering events of light in turbid media is based on the radiative theory. It is assumed that the scattering event of light is independent and has no coherence effects. In addition, we assume that all of the linearly birefringent tissue can be looked on as a uniaxial material with the slow axis (the orientation with higher refractive index) along the direction of the collagen fibers and the fast axis (the orientation with lower refractive index) along the cross section. Scattering is assumed to be caused by the spherical particles that randomly suspend among the birefringent media. The birefringent effect of the considered medium is homogeneous everywhere in the sample, which means that, for different positions in the sample, the birefringent orientation and the birefringent value are the same. The geometry of a multiple scattering event is shown in Figure 1. A narrow pencil beam propagates into a plan-parallel birefringent turbid medium downward along the $z$ axis. In this figure, we assume that the direction of the slow axis of the linear birefringence is along the $x$ axis. Photons are scattered in the medium by spherical particles, therefore Mie theory can be used to describe the scattering events. Diffusely backscattered photons are recorded as a function of $(x, y)$ on the upper surface of the medium.

A Stokes vector $\mathbf{S}_{0}$ describes the polarization state of the incident photon packet. An individual photon packet propagates in the turbid medium until it is scattered by a spherical particle. The transport path length $s$ between two adjacent scatters is randomly sampled according to $s=-\ln (\xi) / \mu_{T}$, where $\xi$ is the random number between 0 and 1 , and $\mu_{T}$ $=\mu_{a}+\mu_{s}$ is the interaction coefficient. At each scattering point, an individual photon packet will drop part of its energy and the loss is represented by $\Delta w=w \times \mu_{a} / \mu_{T}$, where $w$ is the weight of the photon packet before the scattering event. In a scattering event, the photon packet will select a polar angle $\Theta$ and an azimuthal angle $\phi$ to decide the orientation of the next step. The angle $\phi$ is positive for a counterclockwise rotation. Once the polar angle $\Theta$ and the azimuthal angle $\phi$ are known, the Stokes vector of the photon packet in the new local coordinate system is given by

$$
\mathbf{S}^{\prime \prime}=\mathbf{M}(\Theta) \mathbf{R}(\phi) \mathbf{S}^{\prime},
$$

where

$$
\begin{gathered}
\mathbf{R}(\phi)=\left[\begin{array}{cccc}
1 & 0 & 0 & 0 \\
0 & \cos (2 \phi) & -\sin (2 \phi) & 0 \\
0 & \sin (2 \phi) & \cos (2 \phi) & 0 \\
0 & 0 & 0 & 1
\end{array}\right], \\
\mathbf{M}(\Theta)=\left[\begin{array}{cccc}
a(\Theta) & b(\Theta) & 0 & 0 \\
b(\Theta) & a(\Theta) & 0 & 0 \\
0 & 0 & d(\Theta) & -e(\Theta) \\
0 & 0 & e(\Theta) & d(\Theta)
\end{array}\right] .
\end{gathered}
$$

$\mathbf{S}^{\prime}$ and $\mathbf{S}^{\prime \prime}$ are the Stokes vectors before and after the scattering event, respectively. $\mathbf{R}$ is the rotation matrix that connects the two Stokes vectors that express the same polarization state of the photon packet in two different reference planes. One reference plane coincides with another after it rotates an angle $\phi$ around the propagation direction of the photon packet. $\mathbf{M}$ is the single scattering matrix deduced from Mie theory, which is expressed in detail in Appendix A.

The polar angle $\Theta$ and the azimuthal angle $\phi$ are not uniformly distributed between $[0, \pi]$ and $[0,2 \pi)$. Sampling of $\Theta$ and $\phi$ depends on the probability density function (PDF) of $\Theta$ and $\phi$ that is a function of the incident Stokes vector

$$
\begin{gathered}
\mathbf{S}=\left[\begin{array}{l}
S_{0} \\
S_{1} \\
S_{2} \\
S_{3}
\end{array}\right] \\
\rho(\Theta, \phi)=a(\Theta)+b(\Theta)\left[S_{1} \cos (2 \phi)+S_{2} \sin (2 \phi)\right] / S_{0} .
\end{gathered}
$$

Detailed sampling equations for $\Theta$ and $\phi$ are shown in Appendix B.

The anisotropic background refractive index around the scattering spheres may slightly change the distributions of the polar angle $\Theta$ and the azimuthal angle $\phi$. However, the birefringence value $\delta$ in biological tissue is quite weak (normally less than $1 \times 10^{-2}$ ), ignoring its perturbation on the PDF of $\Theta$ and $\phi$ is reasonable. Therefore, the birefringent effect on the polarization is only considered during the paths of light between scattering events.

A photon propagates a random distance $s$ in a linearly birefringent turbid medium between two adjacent scattering events. The birefringent effect of the considered medium during these discontinuous steps can be looked at as linear retarders with two parameters, $\Delta$ and $\beta$, as shown in Eq. (5) 
Propagation of Polarized Light in Birefringent Turbid Media ...

$$
\begin{gathered}
\mathbf{T}(\Delta, \beta)=\left[\begin{array}{cccc}
1 & 0 & 0 & 0 \\
0 & C_{4} \sin ^{2}(\Delta / 2)+\cos ^{2}(\Delta / 2) & S_{4} \sin ^{2}(\Delta / 2) & -S_{2} \sin (\Delta) \\
0 & S_{4} \sin ^{2}(\Delta / 2) & -C_{4} \sin ^{2}(\Delta / 2)+\cos ^{2}(\Delta / 2) & C_{2} \sin (\Delta) \\
0 & S_{2} \sin (\Delta) & -C_{2} \sin (\Delta) & \cos (\Delta)
\end{array}\right] \\
\text { C } C_{2}=\cos (2 \beta), C_{4}=\cos (4 \beta), S_{2}=\sin (2 \beta), S_{4}=\sin (4 \beta) .
\end{gathered}
$$

$\beta$ is the azimuthal angle of the birefringent slow axis on the $x-y$ plane in the local coordinate system of the propagating photon. $\Delta$ is the retardation, which can be obtained by

$$
\Delta=(\Delta n) \frac{2 \pi s}{\lambda^{\prime}},
$$

where $\Delta n$ is the difference between the maximum and the minimum refractive indices of the plane perpendicular to the propagation direction of the photon packet, $s$ is the step length, and $\lambda^{\prime}$ is the wavelength of light in the sample medium. When the angle $\alpha$ between the slow axis of birefringence and the propagation direction of the photon packet is known, $\Delta n$ can be expressed by

$$
\Delta n=\frac{n_{s} n_{f}}{\sqrt{\left(n_{s} \cos \alpha\right)^{2}+\left(n_{f} \sin \alpha\right)^{2}}}-n_{f} .
$$

The terms $n_{s}$ and $n_{f}$ are the refractive indices along the slow axis and the fast axis of the birefringent medium that satisfy the relationship $n_{s}=n_{f}+\delta$, where $\delta$ is the linear birefringence value.

Having identified the process of a single step of the photon packet in the birefringent turbid medium, we can express the Stokes vector of a photon packet diffusely reflected after it has been scattered $n$ times in the turbid medium

$$
\begin{aligned}
\mathbf{S}_{n}^{\mathrm{bs}}\left(x^{\prime}, y^{\prime} ; \mu_{s}, \mu_{a}, \delta\right) & \\
= & {\left[\mu_{s} /\left(\mu_{a}+\mu_{s}\right)\right]^{n} \times \mathbf{R}\left(\phi_{L}\right) \mathbf{T}\left(\Delta_{n}, \beta_{n}\right) \mathbf{M}\left(\Theta_{n}\right) } \\
& \quad \times \mathbf{R}\left(\phi_{n}\right) \ldots \mathbf{T}\left(\Delta_{1}, \beta_{1}\right) \mathbf{M}\left(\Theta_{1}\right) \mathbf{R}\left(\phi_{1}\right) \mathbf{T}\left(\Delta_{0}, \beta_{0}\right) \mathbf{S}_{0},
\end{aligned}
$$

where $\mathbf{S}_{0}$ and $\mathbf{S}_{n}^{\mathrm{bs}}$ represent the Stokes vectors of the incident and the backscattered photons, respectively; $\mu_{s}, \mu_{a}$ are the scattering and absorption coefficients, respectively; $\delta$ is the linear birefringence value; $\left(x^{\prime}, y^{\prime}\right)$ is the detection point on the upper surface of the turbid medium in the laboratory coordinate; $\left[\mu_{s} /\left(\mu_{a}+\mu_{s}\right)\right]^{n}$ expresses the remaining energy after the photon has been scattered $n$ times; $\mathbf{T}\left(\Delta_{i}, \beta_{i}\right) \quad(i$ $=0,1, \ldots n)$ describes the birefringent effect on the photon packet during each period of path; $\mathbf{M}\left(\Theta_{i}\right)(i=1,2, \ldots n)$ describes each single scattering event during propagation; $\mathbf{R}\left(\phi_{i}\right)(i=1,2, \ldots n)$ is the rotation matrix that connects the reference plane and the scattering plane for each scattering event; $\mathbf{R}\left(\phi_{L}\right)$ is the rotation matrix that transforms the Stokes vector of the photon in the local coordinate system to the Stokes vector in the laboratory coordinate system. In our Monte Carlo simulation, a local coordinate system is propagated together with the Stokes vector of the photon packet.
After each scattering event, the local coordinate system alters according to the polar angle $\Theta$ and the azimuthal angle $\phi$. When a photon packet exits the surface of the sample, an azimuthal angle $\phi_{L}$ will be calculated, from which the Stokes vector can be transformed back to a laboratory coordinate system.

In Monte Carlo simulations, a large number of independent photon packets will be launched to obtain average detection signals. For a certain detection point on the surface of the medium, the Stokes vector for backscattered light should be the sum of the Stokes vectors of all of the photon packets that escaped the medium from this point

$$
\mathbf{S}_{\mathrm{sum}}^{\mathrm{bs}}\left(x, y ; \mu_{s}, \mu_{T}, \delta\right)=\sum_{i=1}^{\mathrm{num}} \mathbf{S}_{i, n}^{\mathrm{bs}}\left(\rho, \phi ; \mu_{s}, \mu_{T}, \delta\right),
$$

where num is the number of photon packets exited from this point.

\section{Development of Double-Scattering Model}

Rakovic and Kattawar demonstrated a relatively simple analytical method, a double-scattering model, to analyze the polarization patterns of diffusely reflected light. ${ }^{10}$ Their analyses revealed that double scattered photons can qualitatively predict the polarization patterns of diffusely backscattered light from turbid media. With this technique, most polarization characteristics of a sample can be effectively expressed through analytic equations. By adding the linear birefringence, we have developed a double-scattering algorithm, which can be used to predict the polarization patterns of light diffusely reflected from birefringent turbid media.

The geometry of a double-scattering event is shown in Figure 2, which is similar to Figure 1 except that only photons that have been scattered twice are counted. The Stokes vectors of the backscattered photons can be written as

$$
\begin{aligned}
\mathbf{S}_{2}^{\mathrm{bs}}(\rho, \phi)= & \mu_{s}^{2} \int_{-h}^{0} \int_{-h}^{0} \frac{d z_{1} d z_{2}}{r^{2}}\left\{\exp \left[-\mu_{T}\left(\left|z_{1}\right|+\left|z_{2}\right|+r\right)\right]\right. \\
& \times \mathbf{T}_{2}\left(\Delta_{2}, \beta_{2}\right) \mathbf{R}(\phi) \mathbf{M}(\pi-\Theta) \mathbf{T}_{1}\left(\Delta_{1}, \beta_{1}\right) \\
& \left.\times \mathbf{M}(\Theta) \mathbf{R}(\phi) \mathbf{T}_{0}\left(\Delta_{0}, \beta_{0}\right) \mathbf{S}_{0}\right\}
\end{aligned}
$$

where $r=\left[\rho^{2}+\left(z-z^{\prime}\right)^{2}\right]^{1 / 2}, \Theta=\tan ^{-1}\left[\left(\rho / z-z^{\prime}\right)\right], h$ is the thickness of the sample.

On the condition that the slow axis of the birefringence is along the $x$ axis in the laboratory coordinate,

$$
\Delta_{0}=\delta \frac{2 \pi z_{1}}{\lambda^{\prime}}, \quad \Delta_{1}=\delta^{\prime} \cdot \frac{2 \pi r}{\lambda^{\prime}}, \quad \Delta_{2}=\delta \cdot \frac{2 \pi z_{2}}{\lambda^{\prime}}
$$




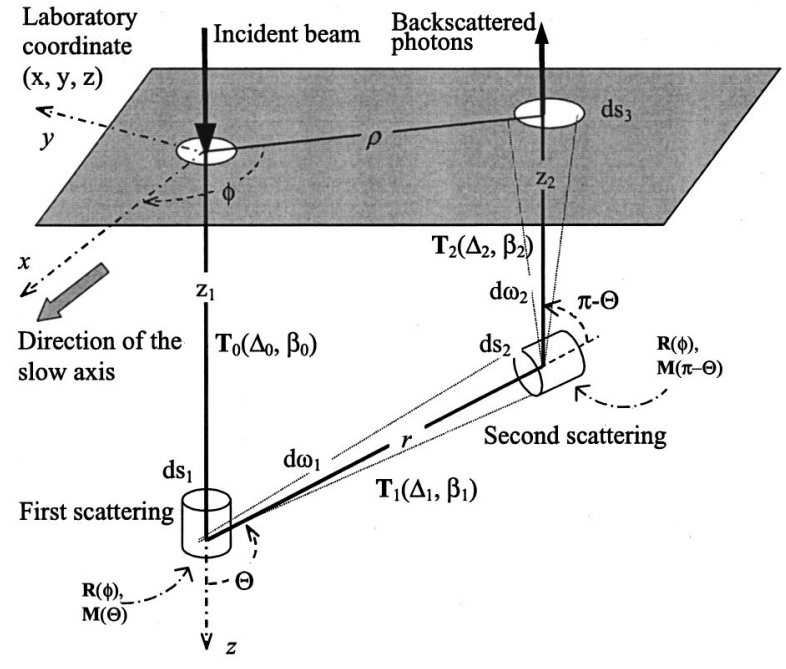

Fig. 2 Geometry of a double-scattering event.

$$
\beta_{0}=\beta_{2}=0, \quad \beta_{1}=\tan ^{-1}\left(\frac{\cos \Theta \sin \phi}{\cos \phi}\right), \quad \delta=n_{s}-n_{f}
$$

The term $\delta^{\prime}$ can be expressed as

$$
\delta^{\prime}=\frac{n_{s} n_{f}}{\sqrt{\left(n_{s} \cos \alpha\right)^{2}+\left(n_{f} \sin \alpha\right)^{2}}}-n_{f},
$$

where

$$
\alpha=\cos ^{-1}(\sin \Theta \sin \phi) .
$$

When the radius of the scattering spheres in the turbid media is small (in the case of Rayleigh scattering), the parameters in matrix $\mathbf{M}$ can be expressed as

$$
\begin{gathered}
a(\Theta)=\frac{3}{16 \pi}\left(1+\cos ^{2} \Theta\right), b(\Theta)=\frac{3}{16 \pi}\left(-1+\cos ^{2} \Theta\right), \\
d(\Theta)=\frac{3}{8 \pi} \cos \Theta, e(\Theta)=0 .
\end{gathered}
$$

By applying the above Eqs. (10)-(15), we can arrive at the analytic expressions of the azimuthal patterns of the 16 Mueller matrix elements of backscattered light as shown in Appen$\operatorname{dix}$ C.

The 16 matrix patterns of backscattered light calculated by our double-scattering model are shown in Figures 3(a) and 3(b) for a birefringent turbid medium and an isotropic turbid medium, respectively, where each map shows the spatial distribution of a Mueller matrix element in gray scale. These figures show comparable results with those simulated by the Monte Carlo algorithm shown in Figure 4, where the scattering coefficient $\mu_{s}$ is $100 \mathrm{~cm}^{-1}$; the absorption coefficient $\mu_{a}$ is $0.1 \mathrm{~cm}^{-1}$; the refractive indices of the background medium are 1.330 and 1.335 along the fast and slow axes, respectively; the wavelength of the light in vacuo is $594 \mathrm{~nm}$. Each matrix element in Figure 3 is a two-dimensional image of the medium surface with the light being injected in the center, $0.2 \times 0.2 \mathrm{~cm}^{2}$ in size.
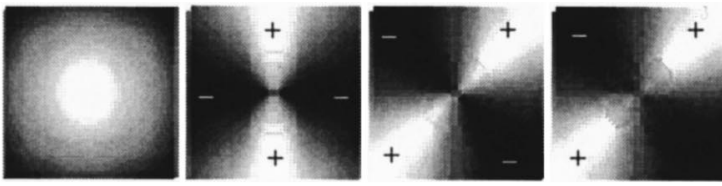

$m_{11}$

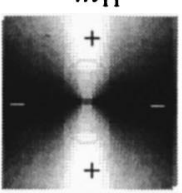

$m_{12}$

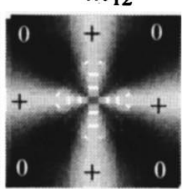

$m_{13}$
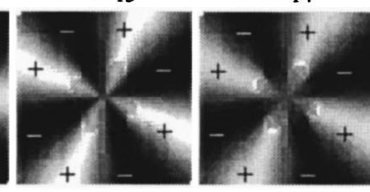

$m_{21}$

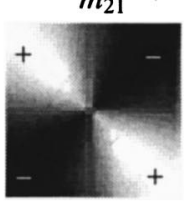

$m_{22}$

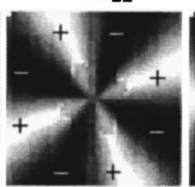

$m_{23}$
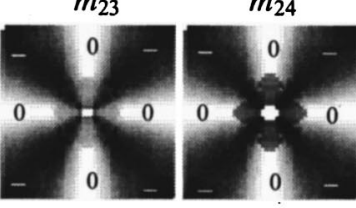

$m_{31}$

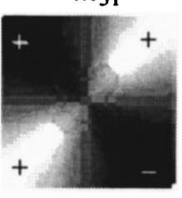

$m_{41}$

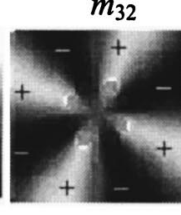

$m_{42}$

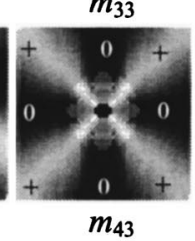

$m_{34}$

(a)
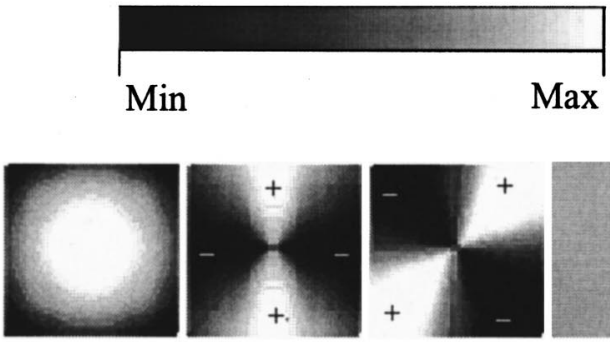

$m_{12}$
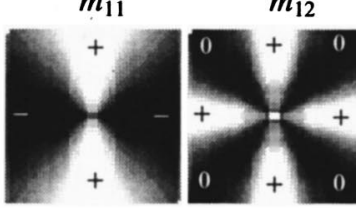

$m_{13}$

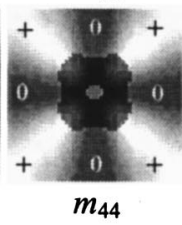

$m_{44}$

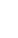

.

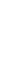



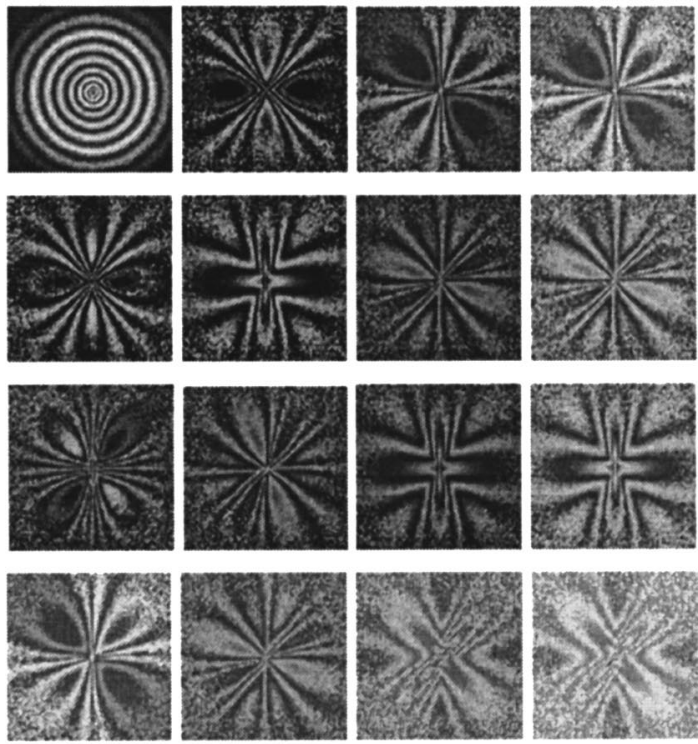

(a)

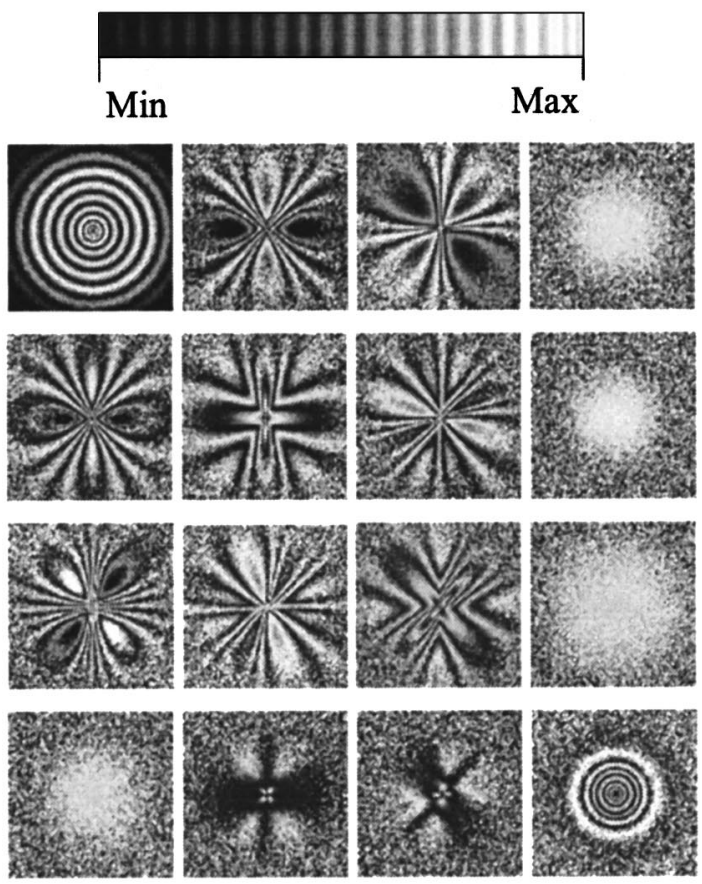

(b)

Fig. 4 Monte Carlo simulated Mueller matrices of light backscattered from (a) a slab of linearly birefringent turbid medium and (b) a slab of isotropic turbid medium. The slow axis of the birefringent medium is along the $x$ axis in the laboratory coordinate. Size of each map is $0.06 \times 0.06 \mathrm{~cm}^{2}$

\section{Results}

\subsection{Mueller Matrix Patterns}

Monte Carlo simulated Mueller matrix patterns of light backscattered from a linearly birefringent turbid medium and an isotropic turbid medium are shown in Figures 4(a) and 4(b), respectively, where each map shows the spatial distribution of a Mueller matrix element in banded gray scale. The slow axis of the birefringent medium is along the $x$ axis in the laboratory coordinate. The birefringence value $\delta$ is $1.0 \times 10^{-3}$. The refractive indices of the birefringent medium along the slow axis and the fast axis are 1.331 and 1.330 , respectively. For the isotropic sample with no birefringent effect, the refractive index of the medium is 1.330 . Other parameters of the two media are the same: the radius of scattering spheres is 350 $\mathrm{nm}$; the refractive index of scatterers is 1.57 ; the wavelength of the light in vacuo is $594 \mathrm{~nm}$; the absorption coefficient $\mu_{a}$ is $1 \mathrm{~cm}^{-1}$; the scattering coefficient $\mu_{s}$ is $90 \mathrm{~cm}^{-1}$; the thickness of the medium slab is $0.2 \mathrm{~cm}$; the anisotropic factor $g$ is calculated to be 0.9; and the size of each picture in Figure 4 is $0.06 \times 0.06 \mathrm{~cm}^{2}$. The main characteristic of every Mueller matrix element in Figure 4 is similar to that of the correspondent map in Figure 3, including the shape as well as the distribution of positive and negative areas. The small discrepancies between Figures 3 and 4 result mainly from the multiple scattering events in the turbid medium that cannot be expressed through the double-scattering model.

The linear birefringence in turbid media variously alters the polarization states of photons according to their trajectories of propagation. Moreover, the sampling of scattering direction (expressed by the polar angle $\Theta$ and the azimuthal angle $\phi$ ) in the single scattering event is a function of the polarization state of the photon being scattered, as shown in Eq. (4). Therefore, the linear birefringence in the turbid medium leads to changes in the spatial intensity distributions of the Mueller matrices of diffusely reflected light. The variations of seven elements, $m_{14}, m_{24}, m_{34}, m_{41}, m_{42}, m_{43}$, and $m_{44}$, among the 16 Mueller matrix elements are most obvious because the transformation between circular polarization state and linear polarization state in the turbid medium is enhanced by the linear birefringence. The shapes and contrasts in the patterns of these seven elements are highly dependent on the birefringence value $\delta$ as well as the orientation of birefringence. With the parameters in Figure 4, the shapes of $m_{14}$, $m_{24}, m_{34}$, and $m_{44}$ are similar to those for $m_{13}, m_{23}, m_{33}$, and $m_{43}$, respectively, though the contrasts in the patterns are different.

It is expected that the pattern of the matrix element $m_{44}$ is no longer rotationally symmetric but depends on the azimuthal angles on the $x-y$ plane in the laboratory coordinate. Because of the direction of the birefringence, the loss of the degree of circular polarization (DOCP) depends on the trajectory of individual photon. The statistical distribution of the change in circular polarization in the birefringent turbid medium can be observed from the spatial intensity pattern of DOCP of the backscattered light. For turbid media with the birefringent slow axis orientated along the $x$ axis, $y$ axis, $45^{\circ}$ on the $x-y$ plane, and $z$ axis, the DOCP patterns of the backscattered light have different azimuthal distributions, as shown in Figures 5(a)-5(d), respectively. Where the incident light is right-circularly polarized, the thickness of the medium slab is set to be $0.1 \mathrm{~cm}$; the other parameters are the same as those used for Figure 4. Conversely, the intensity patterns of the backscattered light can provide particular information about the birefringence in the turbid medium for subsurface imaging. 


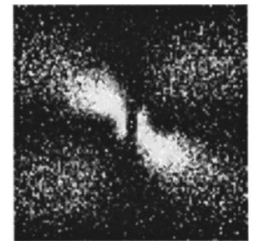

(a)

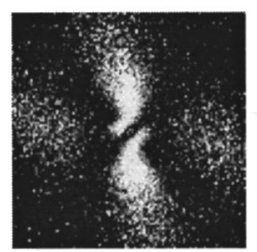

( c)

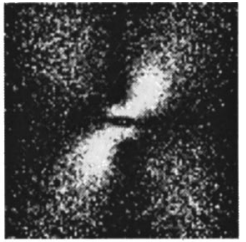

(b)

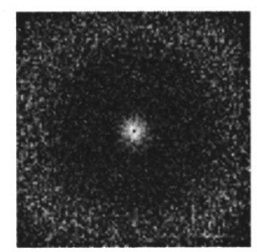

(d)
Fig. 5 DOCP patterns of backscattered light from a linearly birefringent turbid medium with the slow axis along the (a) $x$ axis, (b) $y$ axis, (c) $45^{\circ}$ angle on the $x-y$ plane, and (d) $z$ axis, respectively. The incident light is right-circularly polarized.

\subsection{Birefringent Effect on the Diffuse Reflectance for Unpolarized Incident Light}

We know that linear retarders will not cause any change in the Stokes vector of unpolarized light. However, once unpolarized photons have been scattered by scattering particles, they will present partial polarization states, which depends on the scattering angle in a single scattering event. On the condition that the sample is made of spherical particles suspended in an isotropic medium without the birefringent effect and the incident light is unpolarized, the statistical DOP of the backscattered photons remains zero, even though the spatial distributions of the polarization may present patterns, for instance, the pictures of elements $m_{11}, m_{21}, m_{31}$, and $m_{41}$ shown in Figure 4(b). With the linear birefringence in a turbid medium, the statistical DOP of backscattered photons may no longer be zero, because the linear birefringence destroys the isotropic property of the medium and has different effects on the polarized photons with different trajectories. In Figure 6, we present the DOP of total backscattered light from a linearly birefringent turbid medium for different birefringence values $\delta$, where the Stokes vectors of all the backscattered photo packets are summed up before the simulation of DOP. The

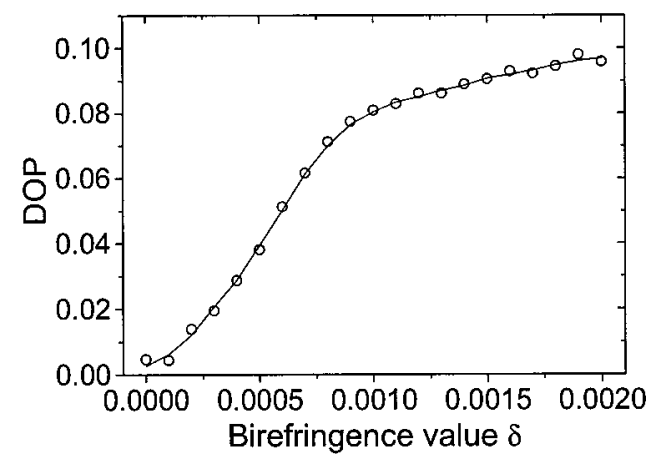

Fig. 6 DOP of backscattered light from a slab of linearly birefringent turbid medium for unpolarized incident light as a function of the birefringence value $\delta$. incident light is unpolarized and the direction of the birefringent slow axis is along the $x$ axis in the laboratory coordinate. $\mu_{a}$ and $\mu_{s}$ are set to be 1 and $90 \mathrm{~cm}^{-1}$, respectively. Under this condition, when the birefringence value $\delta$ is $2 \times 10^{-3}$, the DOP of backscattered light increases to nearly 0.1 .

Because of the discrepancy of the birefringent effect on polarized light with different propagation orientation and different polarization states, and because of the spatial distribution of polarization after single scattering events in the Mie regime, for unpolarized incident light, the intensity distribution of the diffuse reflectance from the birefringent turbid media may no longer be circularly symmetric. Instead, it depends on the azimuthal angle as well as the orientation of the linear birefringence in the medium. This is a reasonable explanation for the phenomenon observed in our previous experiments. ${ }^{17}$ In that work, oblique-incidence reflectometry was adopted for measuring the apparent absorption and reduced-scattering coefficients along different directions in the sample according to the orientation of tissue fibers. Because of the linear birefringence caused by muscle fibers, the spatial distributions of the diffuse reflectance were not equal for the probe aligned at $0^{\circ}$ (parallel) and $90^{\circ}$ (perpendicular) to the orientation of the muscle fibers. Therefore, calculations based on diffusion theory demonstrated different optical parameters of chicken breast tissue along different orientations. In that experiment on a chicken breast sample with birefringent effect, when the wavelength of the incident light in vacuo was $450 \mathrm{~nm}$, the detected absorption coefficient $\mu_{a}$ for the probe aligned at $0^{\circ}$ was smaller than that with the probe aligned at $90^{\circ}$, while the detected reduced-scattering coefficient $\mu_{s}^{\prime}$ for the probe aligned at $0^{\circ}$ was bigger than that with the probe aligned at $90^{\circ}$. For a turbid medium with a birefringent value equal to 0 (nonbirefringent turbid media), the detected $\mu_{a}$ and $\mu_{s}^{\prime}$ are independent of the orientation of the detection probe. With our Monte Carlo algorithm for birefringent turbid media, we can imitate the method adopted in the experiment. As shown in Figure 7(a), a beam of unpolarized light was obliquely injected upon a linearly birefringent turbid medium, where the direction vector of the beam is $(\sqrt{2} / 2,0, \sqrt{2} / 2)$ in the laboratory coordinate; the birefringence value $\delta$ is 0.01 ; the refractive index of the medium along the fast axis is 1.330 ; the refractive index of scatterers is 1.57 ; the radius of the scattering spheres is $280 \mathrm{~nm}$; and the wavelength of light in vacuo is 450 $\mathrm{nm}$. The slow axis of the birefringence was set to be along the $x$ axis and the $y$ axis in the laboratory coordinate, respectively. After multiple scattering events, photons exiting the medium from the area near the $x$ axis were collected for measurement of the spatial distribution of diffuse reflectance along the $x$ axis. Through calculation, $\Delta x_{1}$ is $0.1 \mathrm{~cm}$ and $\Delta x_{2}$ is $0.19 \mathrm{~cm}$ for the slow axis of birefringence along the $x$ axis and $y$ axis, respectively, as shown in Figure 7(b). Using the algorithm developed by Wang and Jacques, ${ }^{18}$ we calculated the apparent absorption coefficient $\mu_{a}$ and reduced-scattering coefficient $\mu_{s}^{\prime}$ for the sample with the birefringent slow axis along the two orientations, respectively. When the slow axis is along the $x$ axis, the absorption coefficient $\mu_{a}$ is $0.13 \mathrm{~cm}^{-1}$ and the reduced-scattering coefficient $\mu_{s}^{\prime}$ is $14.10 \mathrm{~cm}^{-1}$. When the slow axis is along the $y$ axis, $\mu_{a}$ is $0.25 \mathrm{~cm}^{-1}$ and $\mu_{s}^{\prime}$ is 6.98 $\mathrm{cm}^{-1}$. It is obvious that when the detection points aligned at $0^{\circ}$ with respect to the slow axis, the simulated $\mu_{a}$ and $\mu_{s}^{\prime}$ are 


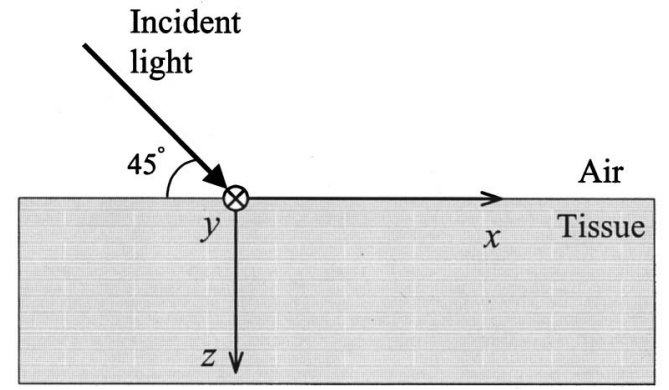

(a)

- The slow axis of the birefringence is along the $x$-axis. - The slow axis of the birefringence is along the $y$-axis.

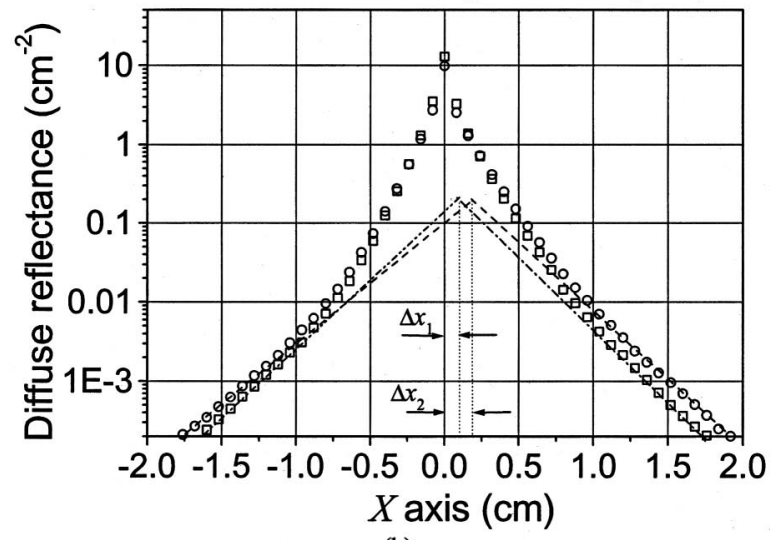

(b)

Fig. 7 (a) Schematic representation of obliquely incident light upon a birefringent turbid medium. The direction vector of the incident light is $[(\sqrt{2} / 2,0, \sqrt{2} / 2)]$ in the laboratory coordinate. For comparison, the slow axis of the birefringent medium is along the $x$ axis and $y$ axis, respectively. (b) Monte Carlo simulated distribution of the diffuse reflectance of light along the $x$ axis on the surface of the turbid medium.

smaller and bigger, respectively, than the simulated results for the detection points aligned at $90^{\circ}$. The relative changes in $\mu_{a}$ and $\mu_{s}^{\prime}$ are in good agreement with the results observed in the experiment.

We know that the refractive index of the medium is higher along the length of fibers than along the cross section. Therefore, the scattering cross section of fibers is anisotropic, which affects the distribution of the light after a scattering event and then changes the result of spatial distribution of the diffuse reflectance. Since the birefringence value $\delta$ in biological tissue is quite weak, it is reasonable to ignore the perturbation of the anisotropic scattering cross section on the spatial distribution of the scattering angle for each scattering event. Therefore, we assume that the main effect of the birefringence on the polarization states comes from phase retardation during the light propagation between adjacent scattering events. Our simulations based on this assumption show that the birefringence effect between adjacent scattering events at least partly explains the phenomenon that linear tissue birefringence alters the diffuse reflectance of unpolarized incident light.

\subsection{Birefringent Effect on the Diffuse Reflectance for Polarized Incident Light}

In general, when a polarized light is projected upon a turbid medium, the DOP of the backscattered photons decreases with

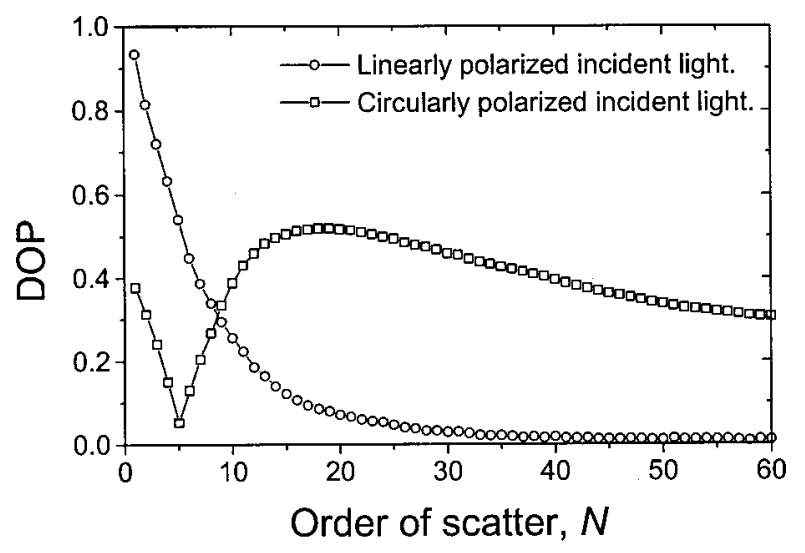

(a)

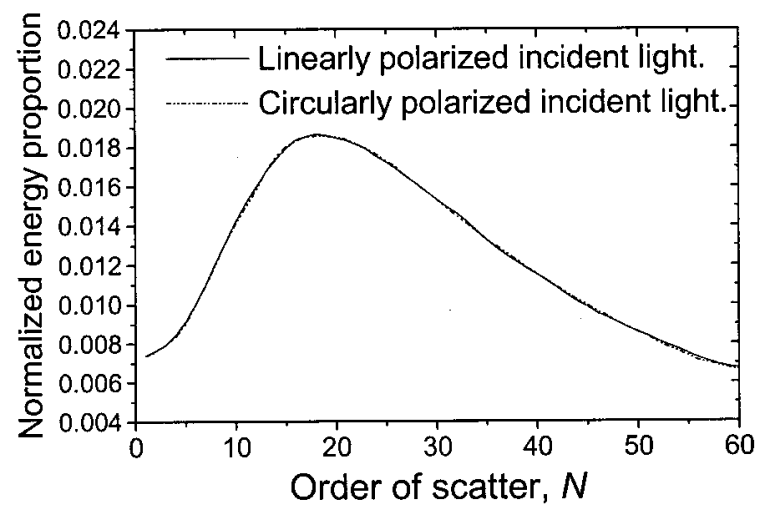

(b)

Fig. 8 (a) DOP and (b) normalized energy proportion of backscattered photons as functions of the order of scatters $N$ for linear and circular incident polarizations.

an increase in the order of scatters during propagation, ${ }^{19}$ which is the reason why backscattered light from a highly scattering turbid medium is partially polarized with a low DOP. We calculated the DOP of photons that have been scattered $N$ ( $N$ is from 1 to 90$)$ times in an isotropic turbid medium for linear and circular incident polarizations, respectively, as shown in Figure 8(a). Figure 8(b) shows the proportion in the backscattered light energy for photons that have been scattered $N$ times, where the wavelength of light in vacuo is $450 \mathrm{~nm}$, the refractive indices of the medium and scattering spheres are 1.33 and 1.57 , respectively; the radius of scattering spheres is $350 \mathrm{~nm}, \mu_{s}$ is $90 \mathrm{~cm}^{-1}$; $\mu_{a}$ is $1 \mathrm{~cm}^{-1}$; and $g$ is calculated to be 0.9 . In order to imitate the actual situation in the experiment, we set the receiving angle of the photon detector to be less than $\cos ^{-1}(0.85)$. For linear incident polarization, the DOP of light backscattered from a high scattering turbid medium comes mainly from photons that have been scattered less than ten times; while for circular incident polarization, the DOP comes mainly from photons that have been scattered 10-30 times. From Figure 8(a) we can see that the circularly polarized photons keep their polarization state better than the linearly polarized photons after multiple scattering. With the above parameters, the size factor $k a$ is calculated to be 6.5 and the scattering events are in the MIE regime. This result coincides with the analysis of Bicout et al. for the multiple scattering on condition that $k a \gg 1 .^{3}$ 


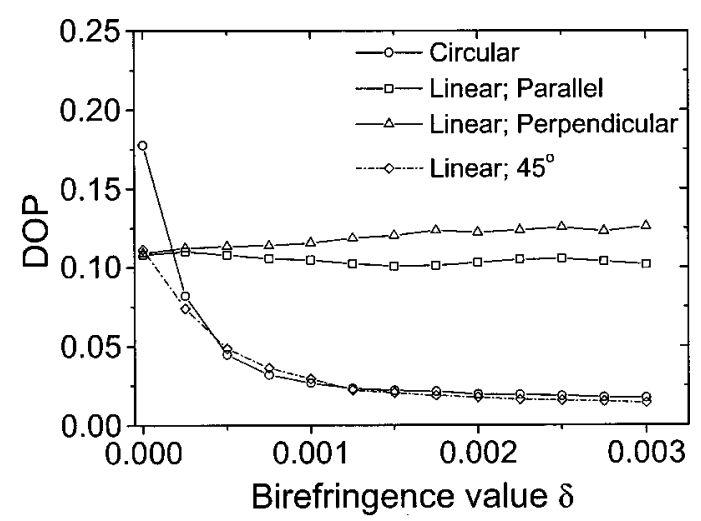

Fig. 9 DOP of backscattered light from a turbid medium with different birefringence value $\delta$. The slow axis of the birefringence is along the $x$ axis in the laboratory coordinate. States of incident light are rightcircularly polarized and linearly polarized with the orientation of polarization parallel, perpendicular and at $45^{\circ}$ angle according to the slow axis of the birefringence, respectively.

Birefringence in a turbid medium alters the polarization states of photons propagating in it. In the Mueller matrix shown in Eqs. (5)-(7), $\beta$ and $\Delta$ are two parameters determined by the step length, the local coordinate of the photon, as well as the orientation and $\delta$ of the linear birefringence. The step length, orientation, and the local coordinate of photon propagating in a highly scattering turbid medium are randomly sampled; therefore $\beta$ and $\Delta$ in the $\mathbf{T}$ matrix between two scattering events are also randomized. For the backscattered photons after multiple scattering in turbid media, the statistical effect of linear birefringence on the initially polarized light is depolarization.

Figure 9 shows the DOP of total backscattered light versus the linear birefringence value $\delta$ for different incident polarizations, where the orientation of the birefringent slow axis is along the $x$ axis in the laboratory coordinate system. Because the DOP of backscattered light for circular incident polarization comes mainly from photons scattered 10-30 times and because of the depolarization of the birefringent effect on the multiply scattered photons in the turbid medium, the DOP of backscattered light tends to approach zero as the $\delta$ increases.

It is interesting that the curves of simulated DOP for backscattered light versus the birefringence value $\delta$ are different for linearly polarized incident light with the polarization axis oriented at different angles on the $x-y$ plane. When the polarization axis for the incident light is oriented at $0^{\circ}$ or $90^{\circ}$, the DOP of diffusely reflected light does not change significantly when $\delta$ increases. While the polarization axis is oriented at $45^{\circ}$, the DOP of diffusely reflected light decreases and tends to approach zero as $\delta$ increases.

We have shown that the DOP of backscattered light for linearly polarized incident light comes mainly from the lowscattered photons, for example, the photons that have been scattered twice. Let us enter

$$
\left[\begin{array}{l}
1 \\
1 \\
0 \\
0
\end{array}\right],\left[\begin{array}{c}
1 \\
-1 \\
0 \\
0
\end{array}\right] \text { or }\left[\begin{array}{l}
1 \\
0 \\
1 \\
0
\end{array}\right] \text {, }
$$

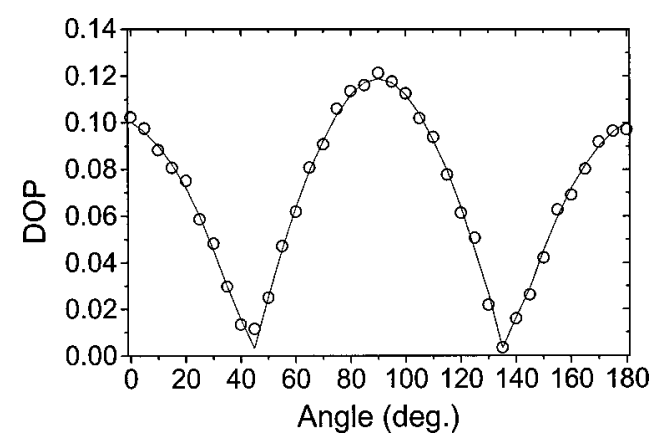

Fig. 10 DOP of backscattered light vs the orientation of the polarization (from $0^{\circ}$ to $180^{\circ}$ ) of the linearly polarized incident light.

respectively, into Eq. (10) for $\mathbf{S}_{0}$. Next, we integrate $\mathbf{S}_{2}^{\mathrm{bs}}$, the Stokes vectors of double scattered photons in the laboratory coordinate system, over the azimuthal angle $\phi \in[0,2 \pi)$ and the radius $\rho \in\left[0, \rho_{0}\right]$ that describe the detected sample surface around the incident point. After reasonable simplification when $\delta$ is large enough, the DOP of all of the double scattered photons for the linearly polarized incident light with the polarization axis oriented along the $x$ axis or $y$ axis tends to be

$$
\begin{aligned}
\operatorname{DOP}_{2}^{\mathrm{bs}}= & \int_{0}^{\rho_{0}} d \rho \int_{0}^{2 \pi} \frac{\sqrt{S_{1}^{2}(\rho, \phi)+S_{2}^{2}(\rho, \phi)+S_{3}^{2}(\rho, \phi)}}{S_{0}(\rho, \phi)} d \phi \\
& \approx \int_{0}^{\rho_{0}} \frac{\int_{-h}^{0} \int_{-h}^{0} \frac{d z_{1} d z_{2}}{r^{2}}\left\{\exp \left[-\mu_{T}\left(\left|z_{1}\right|+\left|z_{2}\right|+r\right)\right] \cdot b(\Theta) b(\pi-\Theta)\right.}{\int_{-h}^{0} \int_{-h}^{0} \frac{d z_{1} d z_{2}}{r^{2}}\left\{\exp \left[-\mu_{T}\left(\left|z_{1}\right|+\left|z_{2}\right|+r\right)\right] \cdot a(\Theta) a(\pi-\Theta)\right.} \\
& \times d \rho \int_{0}^{2 \pi} \cos ^{2}(2 \phi) d \phi
\end{aligned}
$$

which is a constant not equal to zero and independent of $\delta$. When the linear incident polarization is oriented along $45^{\circ}$ on the $x-y$ plane, we can determine by deduction that the DOP of the total double-scattered photons tends to be zero when $\delta$ increases. Moreover, we find that through analytic deduction other low-scattered photons present similar results. For a turbid medium with linear birefringence (the birefringent slow axis is along the $x$ axis in the laboratory coordinate), the DOP of total backscattered light versus the orientation of the polarization (from $0^{\circ}$ to $180^{\circ}$ ) of the incident light is shown in Figure 10. The peak of the DOP appears when the polarization of the incident light is oriented at $0^{\circ}, 90^{\circ}$, or $180^{\circ}$ on the $x-y$ plane while the lowest value appears when the polarization is oriented at $45^{\circ}$ or $135^{\circ}$.

\subsection{Comparison with OCT Results}

Depth-resolved monitoring of tissue birefringence is accurate using polarization-sensitive OCT. With our Monte Carlo algorithm for photon propagation in birefringent turbid media, we can explain the experimental results obtained previously and explore new optical phenomena in fibrous tissues. In order to imitate the actual situation of OCT, we only count the lowscattered photons (order of scatters less than 2) for $\mathbf{S}_{\mathrm{OCT}}^{\mathrm{bs}}$ $=\left[S_{0}, S_{1}, S_{2}, S_{3}\right]^{T}$, the detected Stokes vectors of backscattered light. The wavelength of light in vacuo is $450 \mathrm{~nm} ; \mu_{s}$ is 


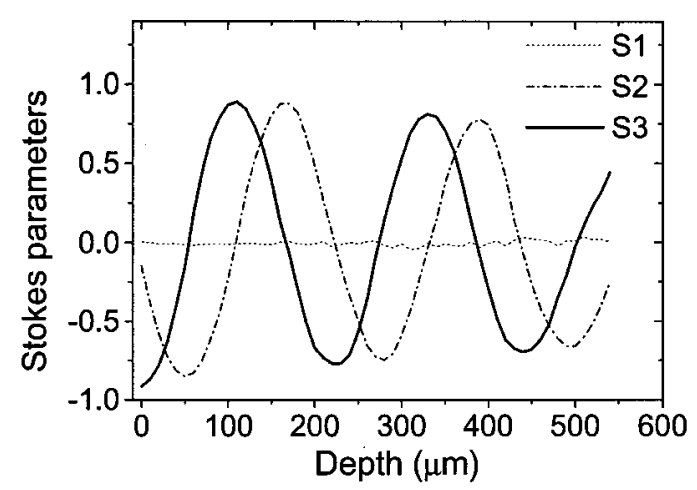

(a)

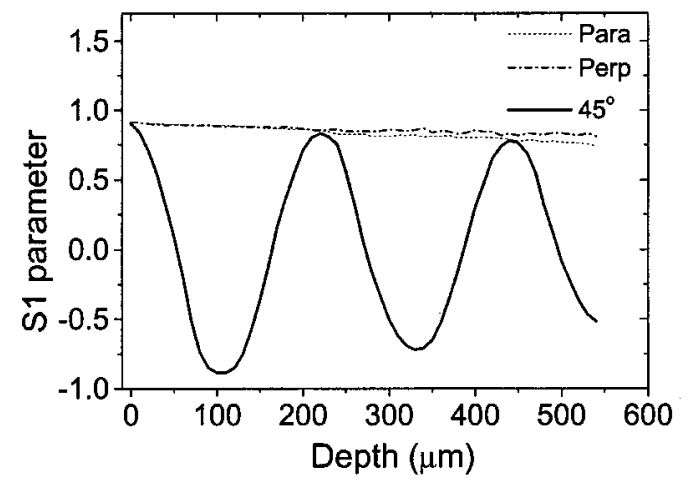

(b)

Fig. 11 Stokes parameters of the light diffusely reflected from different depths in the linearly birefringent turbid medium for (a) right-circular incident polarization and (b) linear incident polarization with orientation parallel, perpendicular and at $45^{\circ}$ with the optical axis. For the comparison with OCT results, only low-scattered photons are counted.

$90 \mathrm{~cm}^{-1} ; \mu_{a}$ is $1 \mathrm{~cm}^{-1} ; g$ is 0.9 ; and the receiving angle of the photon detector is set to be less than $\cos ^{-1}(0.95)$.

Figure 11 shows the simulated Stokes parameters of backscattered light from a birefringent turbid medium versus the depth where the light is reflected. The birefringence value $\delta$ is set to be $1.0 \times 10^{-3}$. For right-circular incident polarization, several periods of $S_{2}$ and $S_{3}$, cycling back and forth between 1 and -1 , are shown in Figure 11(a), which can be explained by the periodic phase retardation caused by the birefringence during the light propagation. Figure 11(b) presents the $S_{1}$ parameter for the linearly polarized incident light with the orientation of polarization either parallel, perpendicular, or at a $45^{\circ}$ angle in respect to the $x$ axis in laboratory coordinate. For the orientation of polarization along the $45^{\circ}$ angle, $S_{1}$ oscillates with increasing depth of reflection because of the birefringent effect in the turbid medium. All of the results in Figure 11 are in good agreement with the OCT measurements obtained by de Boer, Milner, and Nelson. ${ }^{20}$ In their experiments, right-circularly polarized light and three kinds of linear polarized light were adopted to incident upon a birefringent tissue, respectively. Then the Stokes parameters with respect to the depth were measured. The profiles of Stokes parameters, $S_{2}, S_{3}$, for right-circularly polarized incident light, and $S_{1}$ for linearly polarized incident light orientated at the $45^{\circ}$ angle all presented oscillations with increasing sample depth as expected for a linear birefringent sample.

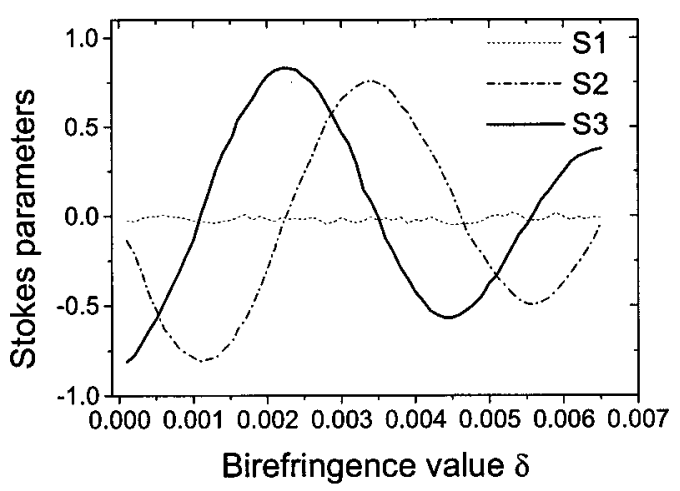

(a)

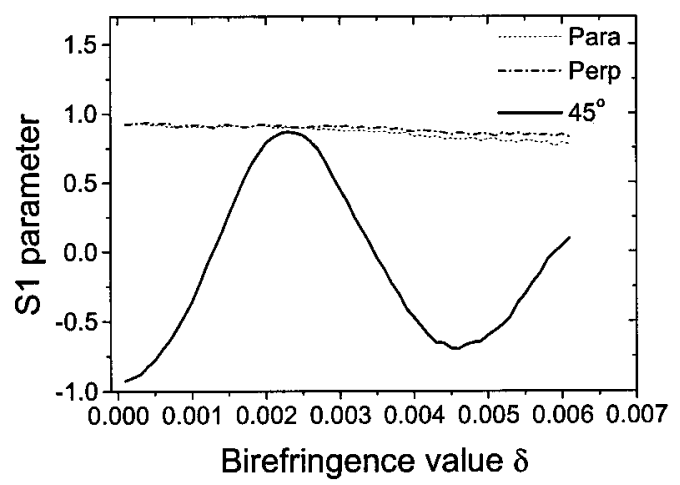

(b)

Fig. 12 Stokes parameters of the backscattered light from a turbid medium with different birefringence value $\delta$ for (a) right-circular incident polarization and (b) linear incident polarization with the orientation parallel, perpendicular, and at $45^{\circ}$ with the optical axis. For comparison with OCT results, only low-scattered photons are counted.

Figures 12(a) and 12(b) show the Stokes parameters of the backscattered light changing with the intensity of birefringence value $\delta$ for right-circular and linear incident polarizations, respectively, where only the photons reflected from a certain depth $(0.05-0.06 \mathrm{~cm})$ in the turbid medium are counted.

\section{Conclusions}

Tissue birefringence caused by fibrous structures is a common phenomenon in biological samples. For polarization-sensitive optical imaging of biological tissue, detailed study of the polarization characteristics of birefringent turbid media is necessary. Based on some reasonable simplification, the Monte Carlo algorithm for the simulation and analysis of the multiple scattering events of polarized light in linearly birefringent turbid media is developed. Simulated spatial patterns of Mueller matrices, spatial patterns of DOP, diffuse reflectance, DOP and Stokes parameters of backscattered light for different birefringent parameters of media as well as different incident polarizations are presented and analyzed in this paper. Some of the results are in good agreement with the phenomenon observed in former experiments, which provides a theoretical basis and explanation for the experimental study of the polarization characteristics of birefringent tissues. 
The Monte Carlo algorithm presented in this paper is based on a specific model, which outlines Mie scattering of light by spherical scatterers in a homogeneous background birefringent medium. The general trends of the results obtained in this paper illustrate the general nature of polarized light behavior in a scattering birefringent medium but do not specifically match the behavior of a specific tissue. The theoretic study presented should contribute to the understanding of the essential physical processes of polarized light propagation in birefringent tissues. Some of the polarization characteristics of backscattered light from birefringent turbid media are observed for the first time, which can provide potential methods for use in actual subsurface tomography of fibrous tissues. In real tissues, the characteristics of birefringence and the scatters are much more complicated. The birefringent orientation and the birefringent value may be different for the various layers in the tissue. When this is the case, the assumption in this paper that the birefringent effect of the considered medium is homogeneous will not hold. However, by using the method introduced in this paper and by dividing the sample into many layers according to the birefringent effect, we can still study those birefringent tissues with complicated structures. ${ }^{21}$ Because of the nature of the Monte Carlo simulation, coherent phenomena cannot be modeled using the method presented in this paper. However, this simulation of polarized light propagation in birefringent turbid media can be applied in the noncoherent regime or in cases where coherent effects are removed.

\section{Acknowledgments}

This study was sponsored in part by National Institutes of Health Grant Nos. R01 CA71980 and R21 CA83760, National Science Foundation Grant No. BES9734491, and Texas Higher Education Coordinating Board Grant No. 0005120123-1999.

\section{Appendix A}

Based on the Mie theory, the single scattering matrix is expressed as

$$
\mathbf{M}(\Theta)=\left[\begin{array}{cccc}
a(\Theta) & b(\Theta) & 0 & 0 \\
b(\Theta) & a(\Theta) & 0 & 0 \\
0 & 0 & d(\Theta) & -e(\Theta) \\
0 & 0 & e(\Theta) & d(\Theta)
\end{array}\right],
$$

where $\Theta$ is the scattering polar angle and

$$
\begin{gathered}
a(\Theta)=\frac{1}{2}\left(\left|S_{2}\right|^{2}+\left|S_{1}\right|^{2}\right), \quad b(\Theta)=\frac{1}{2}\left(\left|S_{2}\right|^{2}-\left|S_{1}\right|^{2}\right), \\
d(\Theta)=\frac{1}{2}\left(S_{2} S_{1}^{*}+S_{1} S_{2}^{*}\right), \quad d(\Theta)=\frac{i}{2}\left(S_{2} S_{1}^{*}-S_{1} S_{2}^{*}\right) .
\end{gathered}
$$

$S_{1}(\Theta)$ and $S_{2}(\Theta)$ are obtained from

$$
\begin{aligned}
& S_{1}(\Theta)=\sum_{n=1}^{\infty} \frac{2 n+1}{n(n+1)}\left(a_{n} \pi_{n} \cos \Theta+b_{n} \tau_{n} \cos \Theta\right), \\
& S_{2}(\Theta)=\sum_{n=1}^{\infty} \frac{2 n+1}{n(n+1)}\left(b_{n} \pi_{n} \cos \Theta+a_{n} \tau_{n} \cos \Theta\right) .
\end{aligned}
$$

Parameters in Eq. (A2) are shown as follows:

$$
\begin{gathered}
a_{n}=\frac{S_{n}^{\prime}(y) S_{n}(x)-n_{\mathrm{rel}} S_{n}(y) S_{n}^{\prime}(x)}{S_{n}^{\prime}(y) \zeta_{n}(x)-n_{\mathrm{rel}} S_{n}(y) \zeta_{n}^{\prime}(x)}, \\
b_{n}=\frac{n_{\mathrm{rel}} S_{n}^{\prime}(y) S_{n}(x)-S_{n}(y) S_{n}^{\prime}(x)}{n_{\mathrm{rel}} S_{n}^{\prime}(y) \zeta_{n}(x)-S_{n}(y) \zeta_{n}^{\prime}(x)}, \\
\pi_{n}=\frac{(2 n-1) \cos \Theta}{n-1} \pi_{n-1}-\frac{n}{n-1} \pi_{n-2}, \\
\tau_{n}=n \cos \Theta \pi_{n}-(n+1) \pi_{n-1},
\end{gathered}
$$

where $n_{\text {rel }}=n_{s} / n_{b}\left(n_{s}\right.$ is the refractive index of the scattering spheres and $n_{b}$ is the refractive index of the background medium), $\pi_{1}=1, \pi_{2}=3 \cos \Theta, \tau_{1}=\cos \Theta$, and $\tau_{2}=3 \cos (2 \Theta)$. $S_{n}(z), S_{n}^{\prime}(z), \zeta_{n}(z)$ can be obtained from

$$
\begin{gathered}
S_{n}(z)=\left(\frac{\pi z}{2}\right)^{1 / 2} J_{n+0.5}(z), \\
S_{n}^{\prime}(z)=\left(\frac{\pi}{8 z}\right)^{1 / 2} J_{n+0.5}(z)+\left(\frac{\pi z}{2}\right)^{1 / 2} J_{n+0.5}^{\prime}(z), \\
\zeta_{n}(z)=S_{n}(z)+i C_{n}(z),
\end{gathered}
$$

where

$$
C_{n}(z)=-\left(\frac{\pi z}{2}\right)^{1 / 2} N_{n+0.5}(z)
$$

$$
C_{n}^{\prime}(z)=-\left(\frac{\pi}{8 z}\right)^{1 / 2} N_{n+0.5}(z)-\left(\frac{\pi z}{2}\right)^{1 / 2} N_{n+0.5}(z)
$$

$J_{n+0.5}(z)$ is the Bessel function of the 1st kind (the order is $n+0.5)$ and $N_{n+0.5}(z)$ is the Bessel function of the 2 nd kind (the order is $n+0.5$ ).

\section{Appendix B}

The combined PDF function of the polar angle $\Theta$ and the azimuthal angle $\phi$ is

$$
\rho(\Theta, \phi)=a(\Theta)+b(\Theta)\left[S_{1} \cos (2 \phi)+S_{2} \sin (2 \phi)\right] / S_{0},
$$

where $a(\Theta)$ and $b(\Theta)$ can be calculated from the equation in Appendix A. Polar angle $\Theta \in[0 \ldots \pi]$ and $a(\Theta)$ satisfies the following normalization requirement:

$$
2 \pi \int_{0}^{\pi} a(\Theta) \sin (\Theta) d \Theta=1
$$


Sampling of $\Theta$ can be fulfilled from Eq. (B2). The cumulative distribution function (CDF) function of $\Theta$ can be expressed as

$$
P\{0 \leqslant \theta \leqslant \Theta\}=2 \pi \int_{0}^{\Theta} a(\theta) \sin (\theta) d \theta=\xi,
$$

where $\xi$ is a random number normally distributed between 0 and 1 . When $\Theta$ is known, the azimuthal angle $\phi$ between [0, $2 \pi$ ] can be sampled according to the PDF function

$$
\rho_{\theta}(\phi)=1+\frac{b(\Theta)}{a(\Theta)} \frac{\left[S_{1} \cos (2 \phi)+S_{2} \sin (2 \phi)\right]}{S_{0}} .
$$

Through the accumulation of the above PDF from 0 to $\phi$, CDF of $\phi$ has the expression

$$
\begin{aligned}
P\{0 & \leqslant \varphi \leqslant \phi\} \\
& =\frac{\int_{0}^{\phi}\left\{1+\frac{b(\Theta)}{a(\Theta)} \frac{\left[S_{1} \cos (2 \varphi)+S_{2} \sin (2 \varphi)\right]}{S_{0}}\right\} d \varphi}{\int_{0}^{2 \pi}\left\{1+\frac{b(\Theta)}{a(\Theta)} \frac{\left[S_{1} \cos (2 \varphi)+S_{2} \sin (2 \varphi)\right]}{S_{0}}\right\} d \varphi} \\
& =\frac{1}{2 \pi}\left\{\phi+\frac{b(\Theta)}{a(\Theta)}\left[\frac{S_{1} \sin 2 \phi+S_{2}(1-\cos 2 \phi)}{2 S_{0}}\right]\right\}=\xi,
\end{aligned}
$$

where $\xi$ is a random number normally distributed between 0 and 1.

In order to expedite the simulation, the $\mathrm{CDF}$ results for $\Theta \in[0, \pi]$ and $\phi \in[0,2 \pi]$ are simulated according to Eqs. (B3) and (B5) before launching the first photon packet, where the step sizes of the two angles are both $\pi / 1000$ in our simulation. When $\Theta$ increases from 0 to $\pi$ (or when $\phi$ increases from 0 to $2 \pi$ ), the $\mathrm{CDF}$ of $\Theta$ (or the $\mathrm{CDF}$ of $\phi$ ) increases from 0 to 1 . In each sampling of $\Theta$ or $\phi$, we first obtain a random number $\xi$. Then, we find the correspondent angle $\Theta$ or angle $\phi$ by sorting the CDF result of the angle.

\section{Appendix C}

The expressions of Mueller matrix patterns of backscattered light from birefringent turbid media are much more complicated than those for isotropic turbid media. After simplification, azimuthal functions of the 16 backscattering Mueller matrix elements $m_{i j}(i, j=1,2,3,4)$ can be expressed as

$$
\begin{gathered}
m_{11}=\left|A_{11}(\rho, \delta)\right|, \\
m_{12}=\left|A_{12}(\rho, \delta)\right| \cdot \cos (2 \phi), \\
m_{13}=-\left|A_{13}(\rho, \delta)\right| \cdot \sin (2 \phi), \\
m_{14}=-\left|A_{14}(\rho, \delta)\right| \cdot \sin (2 \phi), \\
m_{21}=\left|A_{21}(\rho, \delta)\right| \cdot \cos (2 \phi), \\
m_{22}=\left|A_{22}(\rho, \delta)\right| \cdot \cos (4 \phi)+\left|B_{22}(\rho, \delta)\right|, \\
m_{23}=-\left|A_{23}(\rho, \delta)\right| \cdot \sin (4 \phi),
\end{gathered}
$$

$$
\begin{gathered}
m_{24}=-\left|A_{24}(\rho, \delta)\right| \cdot \sin (4 \phi), \\
m_{31}=\left|A_{31}(\rho, \delta)\right| \cdot \sin (2 \phi), \\
m_{32}=\left|A_{32}(\rho, \delta)\right| \cdot \sin (4 \phi), \\
m_{33}\left|A_{33}(\rho, \delta)\right| \cdot \cos (4 \phi)-\left|B_{33}(\rho, \delta)\right|, \\
m_{34}=\left|A_{34}(\rho, \delta)\right| \cdot \cos (4 \phi)-\left|B_{34}(\rho, \delta)\right|, \\
m_{41}=-\left|A_{41}(\rho, \delta)\right| \cdot \sin (2 \phi), \\
m_{42}=-\left|A_{42}(\rho, \delta)\right| \cdot \sin (4 \phi), \\
m_{43}=-\left|A_{43}(\rho, \delta)\right| \cdot \cos (4 \phi)+\left|B_{43}(\rho, \delta)\right|, \\
m_{44}=-\left|A_{44}(\rho, \delta)\right| \cdot \cos (4 \phi)+\left|B_{44}(\rho, \delta)\right| .
\end{gathered}
$$

All of the coefficients $A_{i j}$ and $B_{i j}$ are functions of $\rho$ (the source-detector distance) and linear birefringence value $\delta$, which are independent with the azimuthal angle $\phi$.

\section{References}

1. V. Sankaran, K. Schonenberger, J. T. Walsh, Jr., and D. J. Maitland, "Polarization discrimination of coherent propagation light in turbid media," Appl. Opt. 38, 4252-4261 (1999).

2. A. H. Hielscher, J. R. Mourant, and I. J. Bigio, "Influence of particle size and concentration on the diffuse backscattering of polarized light from tissue phantoms and biological cell suspensions," Appl. Opt. 36, 125-135 (1997).

3. D. Bicout, C. Brosseau, A. S. Martinez, and J. M. Schmitt, "Depolarization of multiply scattered waves by spherical diffusers: influence of the size parameter," Phys. Rev. E 49, 1767-1770 (1994).

4. R. R. Anderson, "Polarized-light examination and photography of the skin," Arch. Dermatol. 127, 1000-1005 (1991).

5. S. L. Jacques, M. Ostermeyer, L. Wang, and D. Stephens, "Polarized light transmission through skin using video reflectometry: toward optical tomography of superficial tissue layers," Proc. SPIE 2671, 44-56 (1996).

6. S. G. Demos and R. R. Alfano, "Optical polarization imaging," Appl. Opt. 36, 150-155 (1997).

7. A. H. Hielscher, A. A. Eick, J. R. Mourant, D. Shen, J. P. Freyer, and I. J. Bigio, "Diffuse backscattering Mueller matrices of highly scattering media," Opt. Express 1, 441-453 (1997).

8. B. D. Cameron, M. J. Rakovic, M. Mehrubeoglu, G. W. Kattawar, S. Rastegar, L. V. Wang, and G. L. Cote, "Measurement and calculation of the two-dimensional backscattering Mueller matrix of a turbid medium," Opt. Lett. 23, 485-487 (1998).

9. M. J. Rakovic, G. W. Kattawar, M. Mehrubeoglu, B. D. Cameron, L. V. Wang, S. Rastegar, and G. L. Cote, "Light backscattering polarization patterns from turbid media: theory and experiment," Appl. Opt. 38, 3399-3408 (1999).

10. M. J. Rakovic and G. W. Kattawar, "Theoretical analysis of polarization patterns from incoherent backscattering of light," Appl. Opt. 37, 3333-3338 (1998).

11. G. Yao and L. V. Wang, "Propagation of polarized light in turbid media: simulated animation sequences," Opt. Express 7, 198-203 (2000).

12. S. Jiao, G. Yao, and L. V. Wang, "Depth-resolved two-dimensional Stokes vectors of backscattered light and Mueller matrices of biological tissue measured with optical coherence tomography," Appl. Opt. 39, 6318-6324 (2000)

13. G. Yao and L. V. Wang, "Two-dimensional depth-resolved Muller matrix characterization of biological tissue by optical coherence tomography," Opt. Lett. 24, 537-539 (1999).

14. M. J. Everett, K. Schoenenberger, B. W. Colston, Jr., and L. B. Da Silva, "Birefringence characterization of biological tissue by use of optical coherence tomography," Opt. Lett. 23, 228-230 (1998).

15. J. F. de Boer, T. E. Milner, M. J. C. van Gemert, and J. S. Nelson, 
"Two-dimensional birefringence imaging in biological tissue by polarization-sensitive optical coherence tomography," Opt. Lett. 22, 934-936 (1997).

16. J. M. Schimitt and S. H. Xiang, "Cross-polarized backscatter in optical coherence tomography of biological tissue," Opt. Lett. 23, 1060-1062 (1998).

17. G. Marquez, L. V. Wang, S. Lin, J. A. Schwartz, and S. L. Thomsen, "Anisotropy in the absorption and scattering spectra of chicken breast tissue," Appl. Opt. 37, 798-804 (1998).

18. L. H. Wang and S. L. Jacques, "Use of a laser beam with an oblique angle of incidence to measure the reduced scattering coefficient of a turbid medium," Appl. Opt. 34, 2362-2366 (1995).

19. A. Ambirajan and D. C. Look, "A backward Monte Carlo study of the multiple scattering of a polarized laser beam," J. Quant. Spectrosc. Radiat. Transf. 58, 171-192 (1997).

20. J. F. de Boer, T. E. Milner, and J. S. Nelson, "Determination of the depth-resolved Stokes parameters of light backscattered from turbid media by use of polarization-sensitive optical coherence tomography," Opt. Lett. 24, 300-302 (1999).

21. X. D. Wang and L. V. Wang, "Propagation of polarized light in birefringent turbid media: time-resolved simulations," Opt. Express 9, 254-259 (2001). 